\title{
Noise Pattern Recognition of Airplanes Taking Off: Task for a Monitoring System
}

\author{
Luis Pastor Sánchez Fernández, Oleksiy Pogrebnyak, José Luis Oropeza Rodríguez, \\ and Sergio Suárez Guerra \\ Center for Computing Research, National Polytechnic Institute \\ Av. Juan de Dios Batiz s/n casi esq. Miguel Othon de Mendizabal, Col. Nueva Industrial \\ Vallejo. CP 07738. Mexico City, Mexico \\ \{1sanchez, olek, joropeza, ssuarez\}@cic.ipn.mx
}

\begin{abstract}
This paper presents an original work for aircraft noise monitoring systems and it analyzes the airplanes noise signals and a method to identify them. The method uses processed spectral patterns and a neuronal network feed-forward, programmed by means of virtual instruments. The obtained results, very useful in portable systems, make possible to introduce redundancy to permanent monitoring systems. The noise level in a city has fluctuations between $50 \mathrm{~dB}(\mathrm{~A})$ and $100 \mathrm{~dB}(\mathrm{~A})$. It depends on the population density and its activity, commerce and services in the public thoroughfare, terrestrial and aerial urban traffic, of the typical activities of labor facilities and used machinery, which give varied conditions that must be faced of diverse ways within the corresponding normalization. The sounds or noises that exceed the permissible limits, whichever the activities or causes that originate them, are considered events susceptible to degrade the environment and the health.
\end{abstract}

Keywords: Aircraft, monitoring, noise, pattern, recognition.

\section{Introduction}

The goal of this research stage is to make aircrafts noise signals analysis, that allows creating a recognition method, that will do possible to identify types or class of aircrafts by means of its noise patterns. These types of aircrafts can be of propeller, turbojet and reaction. On the other hand, it is possible to classify the aircrafts as long reach (high power), medium reach (medium power) and short reach (low power).

Committees of Aerial Transport and Environmental propose an aircraft classification based on the level of noise emission. The proposed common classification of aircraft is based on the principle that the aircraft operator should pay a fair price that should be proportional to its noise impact, independently of the weight of the aircraft or of the transport service rendered. Such data would make it possible to recognize the environmental merits of larger aircraft, even if these aircraft are noisier in absolute terms when compared to smaller aircraft [1].

Much of this work involves the collection and analysis of large amounts of aircraft noise data from the Noise and Track Keeping systems (NTK) installed at airports. 
Like any other measured quantity, aircraft noise measurements are subject to some uncertainty, which can influence the quality of the final measured result [2].

The uncertainty contributions for a typical noise study can be considered in two groups. The first group includes the components of uncertainty associated with the measurement of aircraft noise at a particular monitoring location. The second group includes the components of uncertainty associated with any subsequent data analysis that may be carried out [2], [3], [4]. The overall accuracy of any type of measurement is limited by various sources of error or uncertainty. Components of uncertainty can essentially be classified as either random or systematic in nature. When making a series of repeated measurements, the effect of the former is to produce randomly different results each time, which are all spread or scattered around an average (mean) value. In contrast, systematic components of uncertainty cause the measurement to be consistently above or below the true value. For example, when measuring the time with a watch that has been set 1 minute slow, there will be a systematic error (or bias) in all the measurements. In a well-designed measurement study, the systematic components of uncertainty should generally be smaller than the random components [2], [5]. Possible sources of uncertainty for aircraft noise measurements include not only the noise instrumentation itself, but also variations in the noise source and propagation path, meteorological variations, the local environment at the measurement site, and also any variance due to data sampling - all of these individual uncertainty components can influence the quality of the final measured result [1]. An internationally accepted procedure for combining and expressing measurement uncertainties is given in the ISO Guide to the Expression of Uncertainty in Measurement [6], [7], [8].

\section{Diagrams and Description of Typical Architecture of an Aircraft Noise Monitoring Station [9], [10]}

Generally, a noise monitoring complex system detects, identifies and analyses the noise produced by arriving and departing aircrafts. The Fig. 1 presents a typical architecture of aircraft noise monitoring stations. The noise monitoring system (NMS) measures aircraft noise according to defined criteria. The first step of the system is the collection of the detected aircraft noise, the second the attribution of the noise to a specific aircraft movement. To perform the correlation of the aircraft noise, additional information is necessary, which will be described later.

\subsection{EMU - Environmental Monitoring Unit}

The EMU consists of: a) a digital microphone; b) a local unit for data backup; c) a modem for transmitting data to the central processing system.

\subsubsection{Microphone Unit}

Each unit is mounted at the end of a mast and equipped with a digital microphone, an anti-wind and bird guard and a lightning arrestor. The microphone captures the analogue noise signal and performs the critical conversion of the signal immediately at the microphone head, and transmits the noise data in digital form to the EMU's 

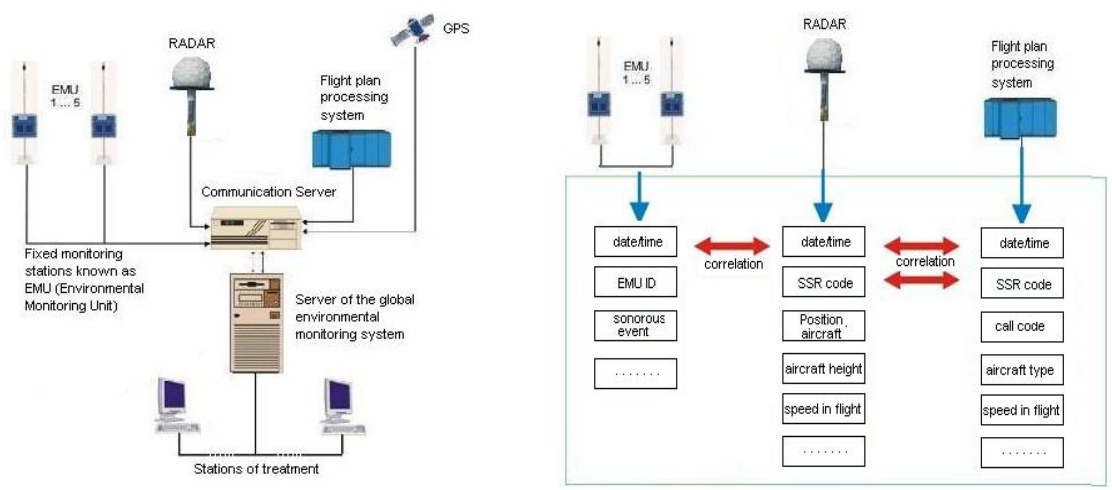

Fig. 1. Typical architecture of aircraft noise monitoring stations and correlation principle

electronics. The immediate conversion to a digital signal provides a higher immunity to interference. The unit guarantees an omni directional detection of noise with high reception qualities. The 5 local units are synchronized by the central system GPS clock.

\subsection{Central Processing System}

All the data collected via network or modem from the airport radar, the flight plan processing system and the EMUs are put through to the central processing system which consists of Communication Server and Global Environment Monitoring System (GEMS). The Communication Server collects: a) the noise events of the five EMUs; b) the radar aircraft tracks; c) the flight plans from FDP (Flight Data Processor); d) the GPS (Global Positioning System) to guarantee the synchronization of the noise monitoring system.

The GEMS processes and correlates the data acquired by the Communication Server in order to identify an aircraft that produced a noise event. The essential processing in the GEMS consists of: a) correlating the real time data from the Communication Server; b) archiving the acquired data, and generating reports about individual events, daily, monthly or annual summaries.

Identification of the noise event: The EMU continuously analyses the incoming noise signal to identify the source of noise. By using various detection algorithms it is possible to identify noise generated by an aircraft flying past, known as event. The process of identifying a noise event is based on threshold and time change criteria. The incoming data are noise events, aircraft flight plans and Radar information. The correlation principle is observed in the right side of Fig 1.

\section{Aircrafts Noise Patterns}

The used airplanes noises in this work have been acquired by means of MP201 microphone. It is a good choice for use in IEC61672 class 1 sound level meters and other noise measurements requiring class 1 accuracy [11]. It is a $1 / 2$ " prepolarized 
free-field measurement microphone. The data acquisition card is USB-9233. It is a four-channel dynamic signal acquisition module for making high-accuracy measurements from IEPE sensors. The USB-9233 delivers $102 \mathrm{~dB}$ of dynamic range. The four USB-9233 input channels simultaneously acquire at rates from 2 to $50 \mathrm{kHz}$. In addition, the module includes built-in antialiasing filters that automatically adjust to your sampling rate. The USB-9233 uses a method of A/D conversion known as deltasigma modulation. If the data rate is $25 \mathrm{kS} / \mathrm{s}$, each ADC actually samples its input signal at 3.2 MS/s (128 times the data rate) and produces samples that are applied to a digital filter. This filter then expands the data to 24 bits, rejects signal components greater than $12.5 \mathrm{kHz}$ (the Nyquist frequency), and then digitally resamples the data at the chosen data rate of $25 \mathrm{kS} / \mathrm{s}$. This combination of analog and digital filtering provides an accurate representation of desirable signals while rejecting out-of-band signals. The built-in filters automatically adjust themselves to discriminate between signals based on the frequency range, or bandwidth, of the signal.

In this work, the noise samples were acquired with sampling frequencies of 22050 $\mathrm{Hz}$ (Samples/second: S/s) and $11025 \mathrm{~Hz}$ (S/s), monophonic and during 24 seconds. In general, this interval is greater than to aircraft takeoff time, or greater to the time in which the produced noise affects the zones near an airport. It is possible to extend this sampling time interval ( $>24$ seconds). For a same aircraft, many noise events were acquired, taken for different meteorological conditions, several microphone orientations and background noises. The takeoff direction is always the same one and this reduces the disturbances of Doppler Effect.

\subsection{Aircraft Noise Signals Analysis}

The Fig. 2 and Fig. 3 present examples of some aircrafts noise signals.

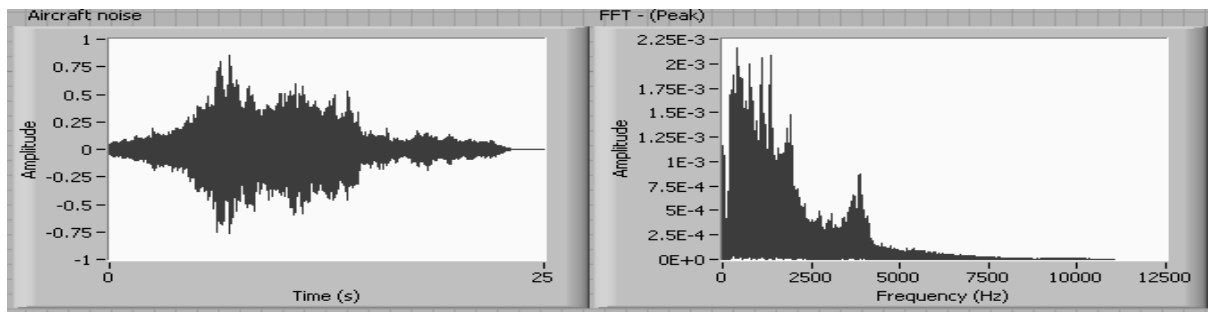

Fig. 2. Noise of Falcon aircraft taking off, with sampling frequency of $22050 \mathrm{~Hz}$

For all used aircraft noises the typical form of the amplitude spectrum is observed from 0 to $5000 \mathrm{Hertz}$, for this reason, in this work was used a sampling frequency of $11025 \mathrm{~Hz}$, in order to reduce the number of taken samples in 24 seconds (264600 samples). The amplitude spectrum has 132300 harmonics, with $\Delta f=0.04167 \mathrm{~Hz}$. In other aircraft noise analyses, the recommended sampling frequency is $25 \mathrm{Ks} / \mathrm{s}$ and D, $\mathrm{C}$ and A-weighting filters. 


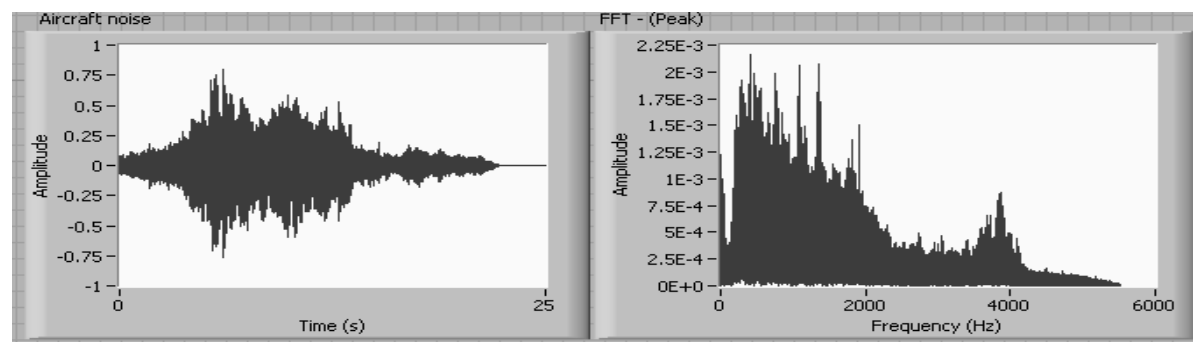

Fig. 3. Noise of Falcon aircraft taking off, with sampling frequency of $11025 \mathrm{~Hz}$

\subsubsection{Reduction of the Spectral Resolution}

It is necessary to reduce the spectral resolution because of the following reasons:

1. The amplitude spectrum has 132300 harmonics and its processing will be very complex.

2. It is only of interest the spectral form.

The following hypotheses are presented:

1. Any reduction method of spectral resolution introduces a tolerance in the initial and final times within the measurement interval of aircraft noise.

For example, a feedforward neural network is trained with one noise pattern which was acquired from zero seconds from the aircraft takeoff until 24 seconds later. In run time, if the aircraft takeoff noise is acquired from 5 seconds until 24 seconds, this time displacement of 5 seconds will affect little the spectral form if its spectral resolution has been reduced.

2. A median filter (moving average filter) creates a typical form of the aircrafts takeoff noises spectrums.

3. The decimation of average spectrum, with a rate $\mathrm{X}$, conserves the spectral form of aircrafts takeoff noises.

\subsubsection{Spectral Estimation}

In the present work is used the Bartlett-Welch method [12] for spectral estimation. The Bartlett method consists on dividing the received data sequence into a number K, of non-overlapping segments and averaging the calculated Fast Fourier Transform.

It consists of three steps:

1. The sequence of $\mathrm{N}$ points is subdivided in $\mathrm{K}$ non overlapping segments, where each segment has length $M$.

$$
\mathrm{x}_{\mathrm{i}}(\mathrm{n})=\mathrm{x}_{\mathrm{i}}(\mathrm{n}+\mathrm{iM}), \mathrm{i}=0,1, \ldots, \mathrm{K}-1, \mathrm{n}=0,1, \ldots, \mathrm{M}-1
$$

2. For each segment, periodogram is calculated

$$
\hat{\mathrm{P}}_{\mathrm{xx}}(\mathrm{f})=\frac{1}{\mathrm{M}}\left|\sum_{\mathrm{n}=0}^{\mathrm{M}-1} \mathrm{x}_{\mathrm{i}}(\mathrm{n}) \mathrm{e}^{-\mathrm{j} 2 \pi \mathrm{fn}}\right|^{2}, \mathrm{i}=0,1, \ldots, \mathrm{K}-1
$$

3. The periodograms are averaged for the $\mathrm{K}$ segments and the estimation of the Bartlett spectral power can be obtained as:

$$
\hat{\mathrm{P}}_{\mathrm{xx}}^{\mathrm{B}}(\mathrm{f})=\frac{1}{\mathrm{~K}}\left|\sum_{\mathrm{i}=0}^{\mathrm{K}-1} \hat{\mathrm{P}}_{\mathrm{xx}}^{(\mathrm{i})}(\mathrm{f})\right|^{2}
$$


The statistical properties of this estimation are the following ones:

The average value is:

$$
\begin{gathered}
\left\langle\hat{P}_{x x}^{B}(f)\right\rangle=\frac{1}{K} \sum_{i=0}^{K-1} \overline{\hat{P}_{x x}^{(i)}(f)}=\overline{\hat{P}_{x x}^{(i)}(f)}=\sum_{m=-(M-1)}^{M-1}\left(1-\frac{|m|}{M}\right) r_{x x}(m) e^{-j 2 \pi f m} \\
=\frac{1}{M} \int_{-1 / 2}^{1 / 2} P_{x x}(a)\left(\frac{\sin \pi(f-\alpha) M}{\sin \pi(f-\alpha)}\right)^{2} d \alpha,
\end{gathered}
$$

where $\frac{1}{M} \frac{\sin \pi(f-\alpha)}{\sin \pi(f-\alpha)}=W_{B}(f)$ it is the frequency characteristic of the Bartlett

window: $\mathrm{w}_{\mathrm{B}}(\mathrm{m})= \begin{cases}\left(1-\frac{|\mathrm{m}|}{\mathrm{N}}\right), & |\mathrm{m}| \leq \mathrm{M}-1 \\ 0 \quad, & |\mathrm{~m}|>\mathrm{M}-1\end{cases}$

The true spectrum is convolutioned with the frequency characteristic of the Bartlett window $\mathrm{w}_{\mathrm{B}}(\mathrm{m})$. Reducing the length of the data window of $\mathrm{N}$ points to $\mathrm{M}=\mathrm{N} / \mathrm{K}$, it results in a window whose spectral wide has been increased by the factor $\mathrm{k}$. Consequently, the frequency resolution has decreased for the factor $k$, in exchange for a variance reduction.

The variance of the Bartlett estimation is:

$$
\operatorname{var}\left[\hat{\mathrm{P}}_{\mathrm{xx}}^{\mathrm{B}}(\mathrm{f})\right]=\frac{1}{\mathrm{~K}^{2}} \sum_{\mathrm{i}=0}^{\mathrm{K}-1} \operatorname{var}\left[\hat{\mathrm{P}}_{\mathrm{xx}}^{(\mathrm{i})}(\mathrm{f})\right]=\frac{1}{\mathrm{~K}} \operatorname{var}\left[\hat{\mathrm{P}}_{\mathrm{xx}}^{(\mathrm{i})}(\mathrm{f})\right]=\frac{1}{\mathrm{~K}} \mathrm{P}_{\mathrm{xx}}^{2}(\mathrm{f})\left[1+\left(\frac{\sin 2 \pi \mathrm{fM}}{\mathrm{M} \sin 2 \pi \mathrm{f}}\right)^{2}\right]
$$

Welch Method [12], [13], [14]: unlike in the Bartlett method, the different data segments are allowed to overlap and each data segment is windowed.

$$
x_{i}(n)=x(n+i D), n=0,1, \ldots, M-1, i=0,1, \ldots, L-1
$$

Where $\mathrm{iD}$ is the point of beginning of the sequence $\mathrm{i}-\mathrm{th}$. If $\mathrm{D}=\mathrm{M}$, the segments are not overlapped. If $\mathrm{D}=\mathrm{M} / 2$, the successive segments have $50 \%$ of overlapping and the obtained data segments are $\mathrm{L}=2 \mathrm{~K}$.

Another modification proposed by Welch to the Bartlet method consists on using a window for the data segments before calculating the periodogram. The result is the "modified" periodogram:

$$
\tilde{\mathrm{P}}_{\mathrm{xx}}^{(\mathrm{i})}(\mathrm{f})=\frac{1}{\mathrm{MU}}\left|\sum_{\mathrm{n}=0}^{\mathrm{M}-1} \mathrm{x}_{\mathrm{i}}(\mathrm{n}) \mathrm{w}(\mathrm{n}) \mathrm{e}^{-\mathrm{j} 2 \pi \mathrm{fn}}\right|^{2}, \mathrm{i}=0,1, \ldots, \mathrm{L}-1
$$

Where $\mathrm{U}$ is a normalization factor for power of the function window and it is selected as:

$$
\mathrm{U}=\frac{1}{\mathrm{M}} \sum_{\mathrm{n}=0}^{\mathrm{M}-1} \mathrm{w}(\mathrm{n})
$$

The Welch estimation of spectral power is the average of these modified periodograms:

$$
\mathrm{P}_{\mathrm{xx}}^{\mathrm{W}}(\mathrm{f})=\frac{1}{\mathrm{~L}} \sum_{\mathrm{i}=0}^{\mathrm{L}-1} \tilde{\mathrm{P}}_{\mathrm{xx}}^{(\mathrm{i})}(\mathrm{f})
$$


The average of the Welch estimation is:

$$
\begin{aligned}
& \left.\qquad \begin{array}{rl}
\mathrm{P} \\
\mathrm{xx}
\end{array}(\mathrm{f})\right\rangle=\frac{1}{\mathrm{~L}} \sum_{\mathrm{i}=0}^{\mathrm{L}-1} \overline{\tilde{\mathrm{P}}_{\mathrm{xx}}^{(\mathrm{i})}(\mathrm{f})}=\overline{\tilde{\mathrm{P}}_{\mathrm{xx}}^{(\mathrm{i})}(\mathrm{f})}=\frac{1}{\mathrm{MU}} \int_{-1 / 2}^{1 / 2} \mathrm{P}_{\mathrm{xx}}(\mathrm{a}) \mathrm{W}(\mathrm{f}-\alpha) \mathrm{d} \alpha \\
& \text { Where } \mathrm{W}(\mathrm{f})=\frac{1}{\mathrm{MU}}\left(\frac{\sin \pi(\mathrm{f}-\alpha) \mathrm{M}}{\sin \pi(\mathrm{f}-\alpha)}\right)^{2}=\frac{1}{\mathrm{MU}} \mathfrak{I}\{\mathrm{W}(\mathrm{n})\}
\end{aligned}
$$

The normalization factor assures that: $\int_{-1 / 2}^{1 / 2} \mathrm{~W}(\mathrm{f}) \mathrm{df}=1$

The variance of the Welch estimation is:

$$
\operatorname{var}\left[\hat{\mathrm{P}}_{\mathrm{xx}}^{\mathrm{W}}(\mathrm{f})\right]=\frac{1}{\mathrm{~L}^{2}} \sum_{\mathrm{i}=0}^{\mathrm{L}-1} \sum_{\mathrm{j}=0}^{\mathrm{L}-1}\left\{\left[\overline{\tilde{\mathrm{P}}_{\mathrm{xx}}^{(\mathrm{i})}(\mathrm{f}) \tilde{\mathrm{P}}_{\mathrm{xx}}^{(\mathrm{j})}(\mathrm{f})}\right]^{2}-\left[\left\langle\hat{\mathrm{P}}_{\mathrm{xx}}^{\mathrm{W}}(\mathrm{f})\right\rangle\right]^{2}\right\}
$$

Why Welch method is introduced?

- Overlapping allows more periodograms to be added, in hope of reduced variance.

- Windowing allows control between resolution and leakage.

The Welch method is hard to analyze, but empirical results show that it can offer lower variance than the Bartlett method, but the difference is not dramatic.

-Suggestion is that $50 \%$ overlapping is used.

In this paper, the data segment of 264600 samples, acquired in 24 seconds, is divided in 24 segments: $K=24$, with $50 \%$ of overlapping, therefore, $L=2 K=48$ overlapped data segments, later is applied the FFT (periodogram) to each segment and they are averaged.

\subsection{Examples of Some Aircrafts Noise Patterns}

1. Normalized noisy spectrum and filtered spectrum

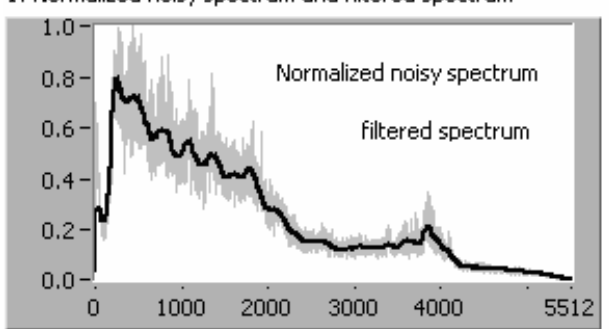

Size of initial spectrum (number of harmonics)

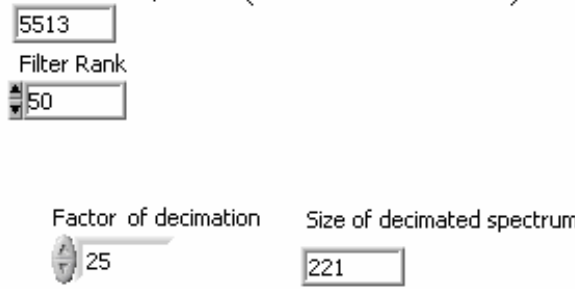

2. Normalized filtered spectrum

3. Decimation of normalized filtered spectrum
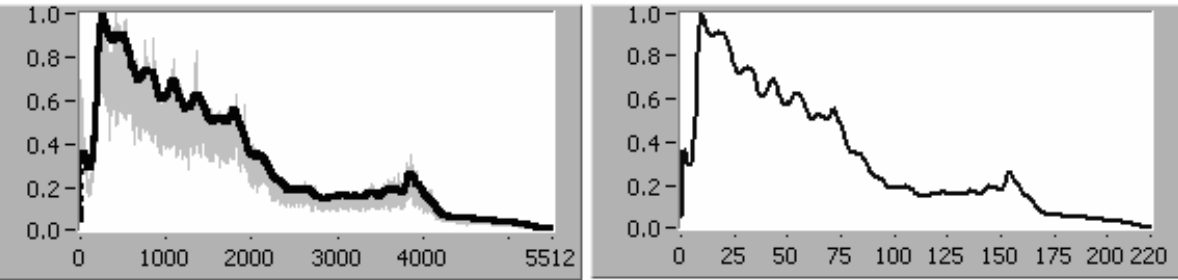

Fig. 4. Example of noise pattern of Falcon aircraft taking off (turbojet) 

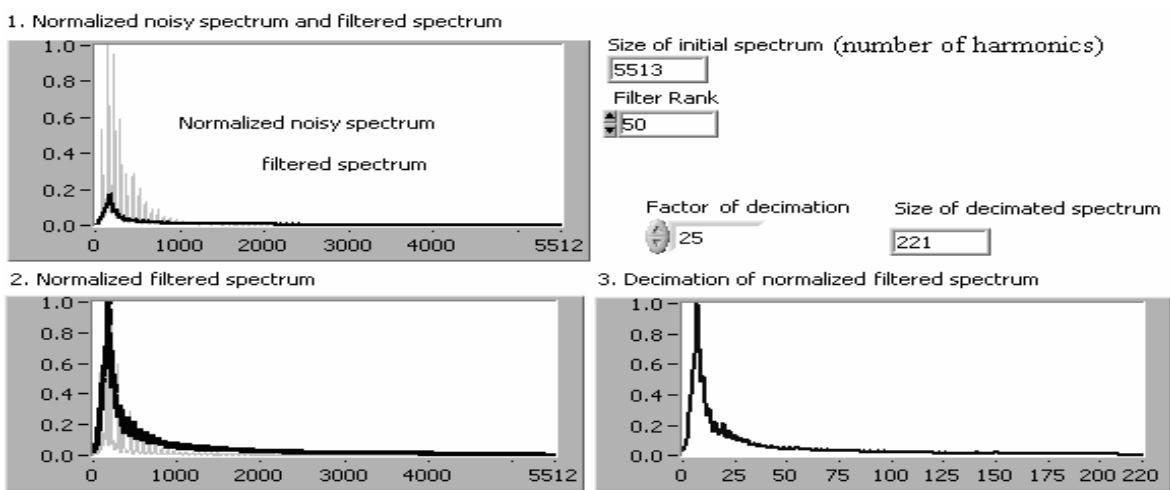

3. Decimation of normalized filtered spectrum

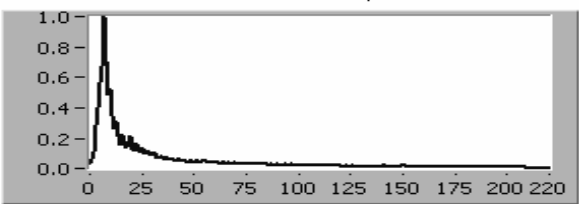

Fig. 5. Example of noise pattern of T6 aircraft taking off (propeller)
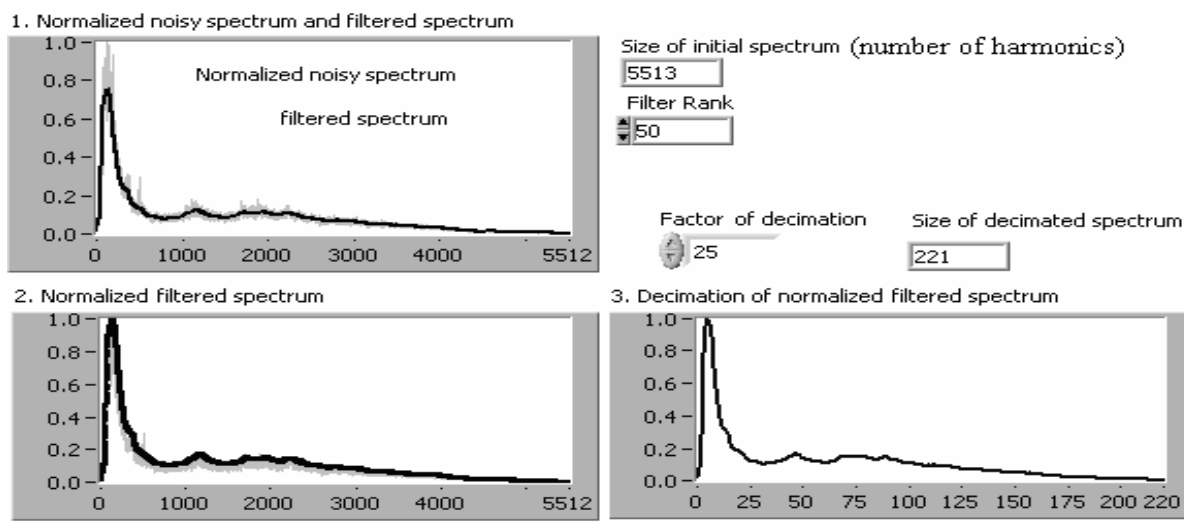

3. Decimation of normalized filtered spectrum

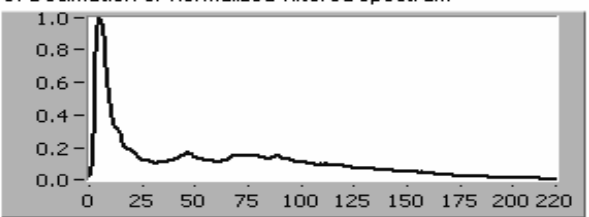

Fig. 6. Example of noise pattern of f Boeing 707 aircraft taking off

In this stage, 16 aircrafts types were tested with 12 patterns by aircraft. In all, the neural network was trained with 192 patterns. Ninety and six patterns were used for testing.

\section{Neural Network}

The neural network has 221 inputs. Every input is a normalized harmonic and some examples were presented in Fig. 4, 5 and 6. The output layer has 16 neurons, corresponding to the 16 recognized aircrafts. After several tests, the neural network was successful with a hidden layer of 14 neurons. The activation functions are tansigmoid. The Fig 7 presents the topological diagram and training performance. The training performance was successful with an error of $1.51281 \mathrm{e}^{-10}$ in 300 epochs. The training finished in 19 minutes in a Pentium IV of $3 \mathrm{GHz}$. 


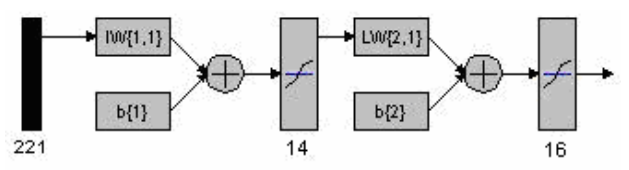

Input: 221 neurons (aircrafts noise harmonics)
Output layer: 16 neurons (aircrafts type)

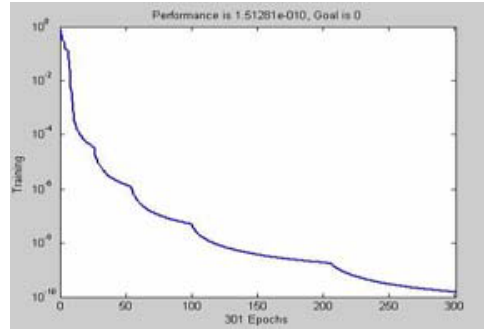

Fig. 7. Neural network topology and training performance

\section{Analysis of Results}

For aircraft noise used in neural network training, the recognition was successful. For an aircraft noise non-used in the training, the neural network non-recognized a specific aircraft and the program presented a message with three aircrafts whose mixed noise patterns have similarity to the acquired noise event (see Fig. 8). Table 1 shows results of a test of aircrafts recognition.

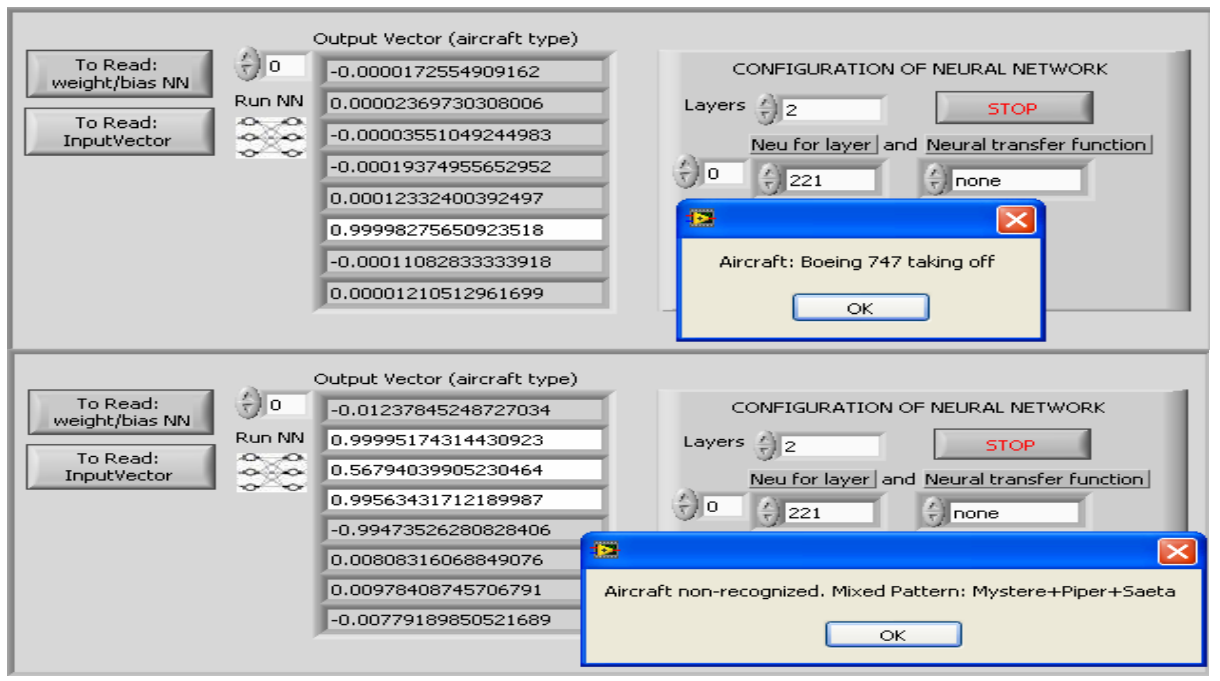

Fig. 8. Example of two tests of patterns recognition and non-recognition

Table 1. Certainty of recognized aircrafts in the first test

\begin{tabular}{|c|c|c|c|}
\hline $\begin{array}{c}\text { Aircrafts } \\
\text { type }\end{array}$ & $\begin{array}{c}\text { Evaluated } \\
\text { Aircrafts }\end{array}$ & $\begin{array}{c}\text { Recognized } \\
\text { aircrafts with } \\
\text { certainty of more } \\
\text { than } \mathbf{8 0} \%\end{array}$ & $\begin{array}{c}\text { Recognized aircrafts with } \\
\text { certainty of mote than } \\
\mathbf{6 0 \%} \text { and less than } \mathbf{8 0} \%\end{array}$ \\
\hline Propeller & 5 & 4 & 1 \\
\hline Turbojet & 17 & 13 & 4 \\
\hline Reaction & 4 & 4 & 0 \\
\hline
\end{tabular}




\section{Conclusions and Future Work}

The presented work tested successfully a methodology to create aircrafts noise patterns. It combines the decrease of spectral resolution, a moving average filter and decimation of average spectrum, this method allows reducing the number of significant harmonics in amplitude spectrum, so that a feedforward neural network with 221 inputs can recognize the aircraft type. The decrease of spectral resolution using the Bartlett-Welch method introduces a tolerance in the initial and final times within the measurement interval of aircraft noise, which produces a better recognition of the patterns when the measurements can have uncertainties. This research tests the feasibility to identify the aircraft that produces a certain noise level, having only noise information. The obtained results make possible to introduce redundancy to permanent monitoring systems of aircraft noise (Fig. 1). These results are very useful in portable systems. The noise intensity and others environmental contamination indicators are calculated by statistical methods using noise time series. The next tests will include more aircrafts types using a distributed and portable system.

\section{References}

1. Kendall, M.: EU proposal for a directive on the establishment of a community framework for noise classification of on civil subsonic aircraft for the purposes of calculating noise charges, European Union (2003)

2. White, S.: Precision of Aircraft Noise Measurements at the London Airports. Environmental Research and Consultancy Department. Civil Aviation Authority. Department for Transport. Ercd Report 0506. ISBN 0-117905-19-4. London (2005)

3. Holding, J.M.: Aircraft noise monitoring: principles and practice. IMC measurement and Control 34(3), 72-76 (2001)

4. Holding, J.M., Sheldon, M.: An expert system for aircraft recognition using acoastics. IMC measurement and Control 34(3), 77-80 (2001)

5. Doebelin, E.O.: Measurement Systems Application and Design. McGraw-Hill, New York (1998)

6. BIPM, IEC, IFCC, ISO, IUPAC, IUPAP, OIML, Guide to the Expression of Uncertainty in Measurement, International Organization for Standardization (ISO), Geneva (1995)

7. Bell, S.: A Beginner's Guide to Uncertainty of Measurement, Measurement Good Practice Guide No. 11(2) National Physical Laboratory (March 2001)

8. Craven, N.J., Kerry, G.: A Good Practice Guide on the Sources and Magnitude of Uncertainty Arising in the Practical Measurement of Environmental Noise, University of Salford (October 2001)

9. Luxembourg Airport Authority: Aircraft noise monitoring stations (2005)

10. Lochard Expanding Environmental Capacity (2006)

11. International Electrotechnical Commission (IEC): Standard IEC61672: Electroacousticssound level meters (2002)

12. Oppenheim, A.V., Schafer, R.W.: Discrete-Time Signal Processing, pp. 311-312. Prentice Hall, Englewood Cliffs, NJ (1989)

13. Welch, P.D.: The Use of Fast Fourier Transform for the Estimation of Power Spectra: A Method Based on Time Averaging Over Short, Modified Periodograms. IEEE Trans. Audio Electroacoust. AU-15, 70-73 (1967)

14. Thompson, S.C.: Spectral Estimation of Digital Signaling Using The Welch Method. Center for Wireless Communications, Department of Electrical and Computer Engineering, University of California at San Diego (2004) 